\title{
Numerische Prozessauslegung zur gezielten Eigenspannungseinstellung in warmmassivumgeformten Bauteilen unter Berücksichtigung von Makro- und Mikroskala
}

\author{
Bernd-Arno Behrens ${ }^{1}$ - Jörg Schröder ${ }^{2}$ Dominik Brands² Kai Brunotte ${ }^{1} \cdot$ Hendrik Wester $^{1}$. \\ Lisa Scheunemann ${ }^{2}$. Sonja Uebing ${ }^{2}$ - Christoph Kock ${ }^{1}$ iD
}

Eingegangen: 22. Januar 2021 / Angenommen: 23. März 2021 / Online publiziert: 20. April 2021

(c) Der/die Autor(en) 2021

\section{Zusammenfassung}

Ziel dieser Arbeit ist die Einstellung eines vorteilhaften Druckeigenspannungsprofils in warmumgeformten Bauteilen durch intelligente Prozessführung mit angepasster Abkühlung aus der Schmiedewärme. Die Machbarkeit und das Potenzial werden an einem Warmumformprozess, bei dem zylindrische Proben mit exzentrischer Bohrung bei $1000^{\circ} \mathrm{C}$ umgeformt und anschließend aus der Schmiedewärme im Wasser abgekühlt werden, aufgezeigt. Vorige Arbeiten zeigen, dass sich Zugeigenspannungen in den derartig umgeformten Proben aus dem Material 1.3505 einstellen. Mittels der vorgestellten mehrskaligen FE-Modelle, wird in dieser Arbeit eine alternative Prozessvariante analysiert, mit der vorteilhafte Druckeigenspannungen anstelle von Zugeigenspannungen durch eine angepasste Abkühlung aus der Umformwärme in den Proben erzeugt werden können. Die angepasste Kühlung wird durch eine partielle Beaufschlagung der Proben mit einem Wasser-Luft-Spray erreicht. Auf diese Weise kann die lokale Plastifizierung durch inhomogene Verzerrungen aufgrund thermischer und umwandlungsinduzierter Effekte beeinflusst werden, um letztlich das Eigenspannungsprofil individuell zu gestalten. Die wissenschaftliche Herausforderung dieser Arbeit besteht darin, unterschiedliche Eigenspannungen in der Oberfläche der Proben zu erzeugen, während die geometrischen und mikrostrukturellen Eigenschaften gleichbleiben. Es wird nachgewiesen, dass eine Beeinflussung der Eigenspannungen und sogar die Umkehr des Spannungsvorzeichens allein durch eine geschickte Prozessführung beim Abkühlen möglich ist.

Christoph Kock

kock@ifum.uni-hannover.de

1 Institut für Umformtechnik und Umformmaschinen, Leibniz Universität Hannover, An der Universität 2, 30823 Garbsen, Deutschland
2 Institut für Mechanik, Abteilung Bauwissenschaften, Universität Duisburg-Essen, Universitätsstraße 15, 45141 Essen, Deutschland 


\title{
Numerical process design for targeted residual stress adjustment in hot bulk formed components taking into account macro- and microscale
}

\begin{abstract}
The aim of this work is the adjustment of an advantageous compressive residual stress profile in hot-formed components by intelligent process control with tailored cooling from the forging heat. The feasibility and potential are demonstrated in a hot forming process in which cylindrical specimen with an eccentric hole are formed at $1000{ }^{\circ} \mathrm{C}$ and subsequently cooled in water from the forging heat. Previous work shows that tensile residual stresses occur in the specimen formed in this way from the material 1.3505. Using the presented multi-scale FE models, an alternative process variant is analysed in this work, where advantageous compressive residual stresses can be generated instead of tensile residual stresses through tailored cooling from the forming heat in the specimen. The tailored cooling is achieved by partially exposing the specimen to a water-air spray. In this way, the local plastification can be influenced by inhomogeneous strains due to thermal and transformation-induced effects in order to customise the resulting residual stress distribution. The scientific challenge of this work is to generate different residual stresses in the surface of the specimen without changing the geometrical and microstructural properties. It is demonstrated that influencing the residual stresses and even reversing the stress sign is possible using smart process control during cooling.
\end{abstract}

\section{Aktuelle Potenziale zur Eigenspannungsbeeinflussung in Schmiedebauteilen}

Eigenspannungen können im Hinblick auf die Betriebsfestigkeit das zyklische Verformungsverhalten verändern, die Rissentstehung fördern oder verzögern, die Rissausbreitung beschleunigen oder verlangsamen und sich vorteilhaft oder nachteilig auf die Ermüdungslebensdauer und die Dauerfestigkeit auswirken [1]. Insbesondere im oberflächennahen Bereich sind Zugeigenspannungen unerwünscht, da sie die Bildung und Ausbreitung von Rissen begünstigen und damit die Ermüdungslebensdauer verringern [2]. Untersuchungen von Löhe et al. zeigen dagegen, dass die Ermüdungslebensdauer von Proben in zyklischen Biegeversuchen durch Druckeigenspannungen, welche mit Oberflächenbehandlungsverfahren wie Kugelstrahlen erzeugt wurden, deutlich erhöht werden kann [3]. In der Arbeit von Pape et al. wird das Potenzial von fertigungstechnisch erzeugten Druckeigenspannungen zur Verzögerung von ermüdungsbedingten Lagerausfällen belegt [4]. Im Allgemeinen sind daher in hochbeanspruchten Bauteilbereichen Druckeigenspannungen möglichst parallel zur höchsten Zugspannung - welche infolge äußerer Lasten auftreten können und rissöffnend wirken - anzustreben. Aus Gleichgewichtsgründen ist die Entstehung von Zugeigenspannungen an anderer Stelle unvermeidlich, sodass diese in weniger beanspruchte Bauteilbereiche verlagert werden sollten.

Aktuelle Fertigungsverfahren zielen bereits auf die Erzeugung eines derartigen vorteilhaften Eigenspannungsprofils ab, wobei die Prozessketten jedoch um zusätzliche zeit-, kosten- und energieintensive Prozessschritte - wie das mechanische Verfahren Kugelstrahlen [5] oder das dem Schmiedeprozess nachfolgende Induktionshärten [6] ergänzt werden müssen [7]. Untersuchungen im Bereich der Warmumformung aus den letzten Jahren zeigen das Potenzial einer Integration der Wärmebehandlung in den Schmiedeprozess für die gezielte Einstellbarkeit der Gefügeeigenschaften bei gleichzeitiger Kosteneinsparung durch die verkürzte Prozesskette [8]. In der Folge erscheint auch die vollständige Integration der Wärmebehandlung zwecks Optimierung der Eigenspannungen in die Schmiedeprozesskette vielversprechend, um die Prozesskosten zu verringern und die Produktqualität zu verbessern. Unklar ist bislang, inwiefern eine Eigenspannungsmodifikation im Bauteil durch die Prozessführung möglich ist, wobei die gewünschten Geometrie- und Gefügeeigenschaften beibehalten werden.

Vor dem Hintergrund des enormen Potentials zur Verbesserung der Bauteileigenschaften sowie auch der Zeitund Kosteneinsparungen durch eine verkürzte Schmiedeprozesskette ist die Motivation, die Entwicklung und Beeinflussbarkeit der Eigenspannungen im Warmumformprozess zu untersuchen. Aufgrund der zahlreichen Einflussparameter, Materialphänomene und deren Wechselwirkungen in der Warmumformung ist eine rein experimentelle Prozessbetrachtung jedoch nicht zielführend. Computergestützte Analysen basierend auf der Finiten Elemente Methode (FEM) bieten dagegen eine wirtschaftliche Möglichkeit zur durchgängigen Prozessanalyse und -auslegung [9]. So lassen sich mit numerischen Methoden verschiedene Prozessszenarien simulieren und dabei auch die zeitlich und örtlich aufgelösten Zustandsdaten während des Prozesses analysieren, was im Experiment nur eingeschränkt möglich ist.

$\mathrm{Zu}$ diesem Zweck wurden bereits in vorherigen Arbeiten mehrskalige FE-Simulationsmodelle für den Werkstoff 1.3505 erstellt und anhand von verschiedenen experimentellen Prozessszenarien mit der Abkühlung von der Warmumformtemperatur in Luft oder Wasser validiert [10]. Es zeigte 
sich, dass in dem untersuchten experimentellen Demonstratorprozess bei einer Abkühlung in Wasser ausschließlich unerwünschte Zugeigenspannungen an der Bauteiloberfläche entstehen. Der Fokus dieser Arbeit liegt auf der gezielten Optimierung des Demonstratorprozesses zur Erzeugung von Druckeigenspannungen im finalen Bauteil durch den Einsatz der entwickelten FE-Simulationsmodelle. Dabei soll die Modifikation der Eigenspannungen vollständig in den Warmumformprozess integriert werden.

In Abschn. 2.1 wird der zu Forschungszwecken entwickelte Demonstratorprozess vorgestellt. Darüber hinaus werden in Abschn. 2.2 werden die Herausforderungen hinsichtlich der Anlagentechnik für eine gesteuerte Abkühlung sowie in Abschn. 2.3 die Anforderungen an die numerischen Modelle aufgezeigt. In Kap. 3 werden die in dieser Arbeit eingesetzten numerischen Modelle präsentiert. Die Ergebnisse der virtuellen Prozessoptimierung werden in Kap. 4 beschrieben und experimentellen Untersuchungen gegenübergestellt. Abschließend folgt in Kap. 5 eine Zusammenfassung des Artikels sowie ein Ausblick auf zukünftige Untersuchungen.

\section{Herausforderungen bei der Modifikation von Eigenspannungen in thermo- mechanisch gekoppelten Prozessen}

\subsection{Demonstratorprozess der Warmumformung}

Für die Entwicklung und Evaluierung der FE-Modelle wurde von den Autoren ein Demonstratorprozess der Warmumformung entworfen [11]. In diesem Prozess werden die in Abb. 1a gezeigten zylindrischen Proben mit einer exzentrischen Bohrung auf $1000^{\circ} \mathrm{C}$ erwärmt und anschließend innerhalb der in Abb. $1 \mathrm{~b}$ dargestellten Thermobox mit dem Umformsimulator DYNSJ5590 um $22 \mathrm{~mm}$ mit $200 \mathrm{~mm} / \mathrm{s}$ gestaucht. Daraufhin erfolgt die Abkühlung der Proben. Für die spätere Untersuchung von Gefüge und Eigenspannungen der Probe werden die Messpunkte (MP) 1 bis 6 auf mittlerer Höhe der Probe entsprechend Abb. 1c definiert. Die exzentrische Bohrung in der verwendeten zylindrischen Probe sorgt für eine inhomogene Spannungsverteilung im gesamten Probenquerschnitt. Auf diese Weise lässt sich ein komplexer Verlauf der Eigenspannungen untersuchen, wie er auch in industriellen Bauteilen vorkommen könnte. Dieser Demonstratorprozess kann genutzt werden, um numerische Modelle durch Abgleich der Eigenspannungswerte zu validieren. So wurde das in Abschn. 3.1 vorgestellte FE-Simulationsmodell über experimentelle röntgendiffraktometrische Untersuchungen für die Abkühlung in Luft oder Wasser validiert. Dabei stellte sich heraus, dass bei der Abkühlung an Luft geringe Druckeigenspannungen im Bereich $<50 \mathrm{MPa}$ und bei der Abkühlung in Wasser, siehe Abb. 1d,
Abb. 1 Ausgangsgeometrie der untersuchten Proben (a), schematische Darstellung der mit Thermoelementen präparierten Probe in einer Thermobox (b), die Messpunkte MP1 bis MP6 auf mittlerer Höhe der Probe (c) sowie röntgenografisch ermittelte Zugeigenspannungen an MP 1 und MP6 nach Wasserabkühlung (d), [11] a

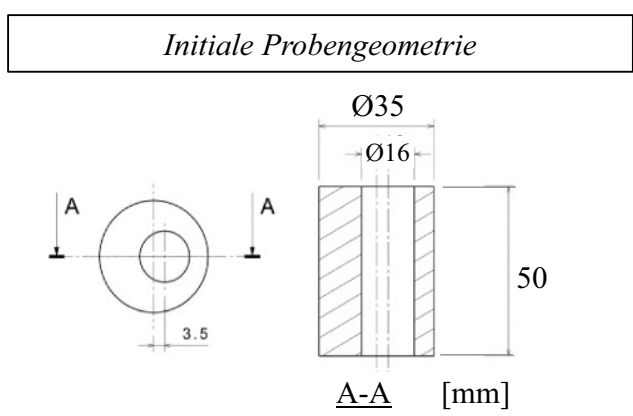

C

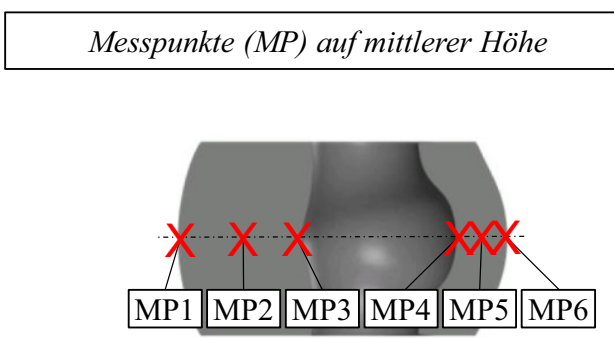

b

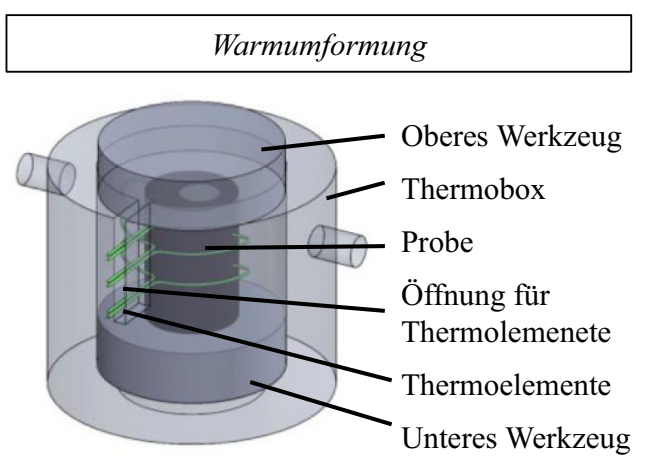

d

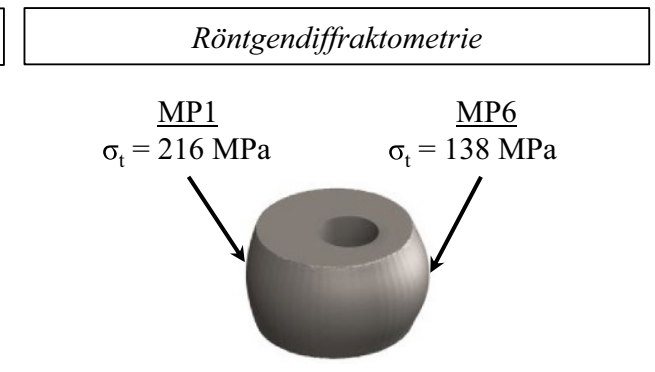


Zugeigenspannungen von $216 \mathrm{MPa}$ an MP1 und $138 \mathrm{MPa}$ an MP6 entstehen [10].

Der Fokus der Arbeit liegt auf einer gezielten Änderung der Zugeigenspannungen in Druckeigenspannungen durch eine Prozessmodifikation. Dabei sollen lediglich die Eigenspannungen verändert werden und die finale Bauteilgeometrie sowie das resultierende Gefüge denen des Referenzprozesses mit Wasserabkühlung entsprechen. Auf diese Weise wird sichergestellt, dass die Veränderung der Eigenspannungen nur im angepassten Prozess und nicht in veränderten Materialeigenschaften begründet ist. Die Eigenspannungen werden somit gezielt durch die Steuerung der lokalen Plastifizierung durch inhomogene Verzerrungen aufgrund von thermischen und umwandlungsinduzierten Belastungen beeinflusst. Neben der generellen Einstellbarkeit vorteilhafter Druckeigenspannungen können abschließend auch die FEModelle hinsichtlich ihrer Einsatzfähigkeit validiert werden.

\subsection{Anlagentechnische Anforderungen}

Während der Abkühlung aus der Umformwärme entwickeln sich die finalen Eigenspannungen im Material in Abhängigkeit von den thermischen und umwandlungsinduzierten Verzerrungen. Die Arbeiten [12,13] und [14] haben gezeigt, dass eine Spraykühlung mit einem Luft-WasserGemisch zur gezielten Steuerung des Temperaturverlaufes nach dem Schmieden geeignet ist. Das Ziel lag darin, eine sogenannte Best-Yield (BY) Behandlung durchzuführen, bei der die gewünschten Werkstoffeigenschaften über den maßgeschneiderten Temperatur-Zeit-Verlauf eingestellt werden. Der Vorteil der Spraykühlung liegt in der individuellen Anpassbarkeit der Spraykegel sowohl örtlich als auch zeitlich. So lassen sich einzelne Düsen auf bestimmte Bauteilpartien ausrichten, um eine lokale Abkühlung zu erzeugen. Darüber hinaus lassen sich die Düsen zeitgesteuert nach Bedarf ein- und ausschalten, um etwa nach dem Här-

a

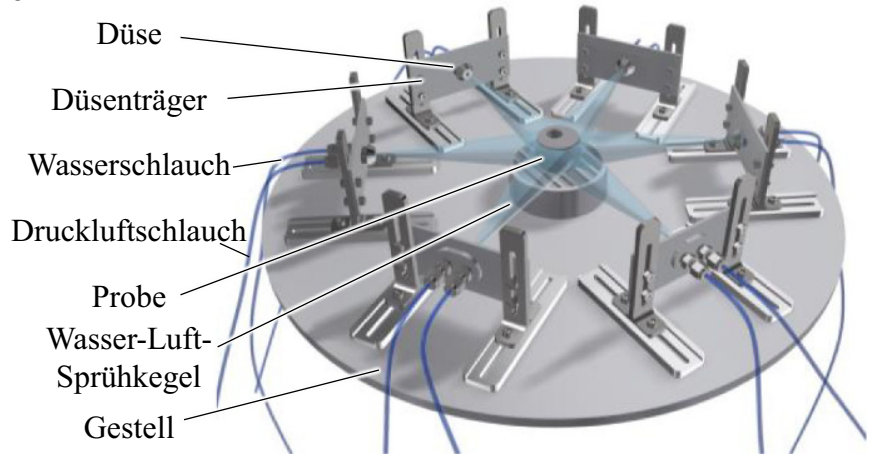

ten einer Bauteiloberfläche Anlasseffekte durch Restwärme aus dem Bauteilkern zu erzeugen.

Für die durchgeführten Studien wird das in Abb. 2a dargestellte Spraykühlsystem verwendet. Dieses arbeitet mit sechs kreisförmig angeordneten Zweistoffdüsen vom Typ XA PR 050 der Bete GmbH. Die Medien Wasser und Luft werden den Düsen mit einem Druck von 0,04 MPa zugeführt. Dabei ergibt sich eine Wasserdurchflussmenge von ca. 0,21/s. Die Magnetventile vor den Düsen werden mit dem digitalen I/O-Modul NI-9375 von National Instruments angesteuert und mit der Software LabView programmiert. Es können entweder alle oder einzelne Düsen aktiviert werden, wobei der Sprühkegel einer Düse etwa $90^{\circ}$ der Umfangsfläche der zylindrischen Proben benetzt. In dieser Arbeit wird die Spraykühlung für kontinuierliche Abkühlvorgänge mit unterschiedlichen Konfigurationen der Düsenanordnung von der Umformtemperatur bis zur Raumtemperatur untersucht.

Der im Sprayfeld entstehende temperaturabhängige Wärmeübergangskoeffizient (HTC, engl.: heat transfer coefficient) für den Werkstoff 1.3505 ist in Abb. 2b im Vergleich zum HTC von 1.3505 bei Abkühlung in Wasser dargestellt. Dieser wurde anhand der in [11] präsentierten experimentell-numerischen Methode ermittelt. Dazu wurden Abkühlversuche von $1000^{\circ} \mathrm{C}$ an zylindrischen Proben mit $\varnothing 40 \mathrm{~mm} \times 50 \mathrm{~mm}$ unter Verwendung von sechs Düsen durchgeführt. Die Temperatur-Zeit-Kurven werden mit einem Thermoelement in der Mitte der Stirnfläche in einer Tiefe von ca. $3 \mathrm{~mm}$ gemessen. Für die Identifizierung des HTC der Spraykühlung wird in simufact.forming ein rotationssymmetrisches 2D-Modell des Zylinders erstellt, analog zu dem in Abschn. 3.1 vorgestellten Modell. Die Abkühlung wird in einer automatisierten Schleife simuliert und unter variierenden HTC-Werten so lange wiederholt, bis mit der Methode der kleinsten Quadrate zwischen den berechneten und experimentellen Temperatur-Zeit-Kurven eine gute Übereinstimmung erzielt wird. Anhand der

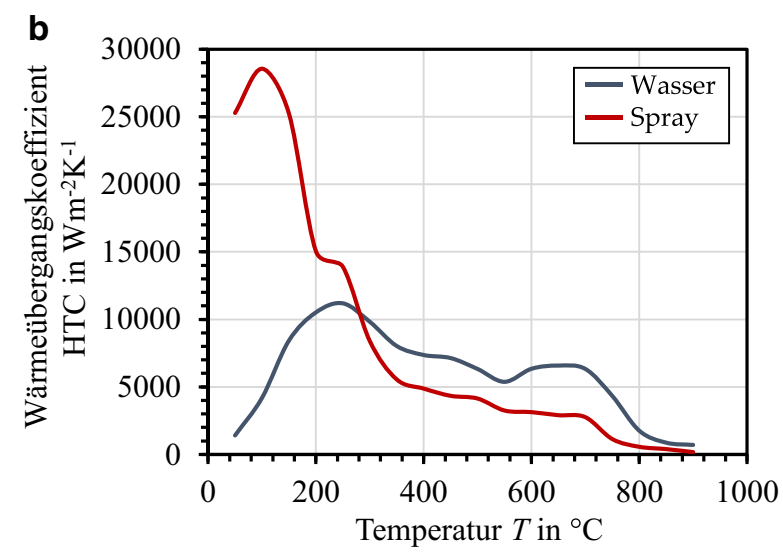

Abb. 2 Wasser-Luft-Spraykühlung für maßgeschneiderte Kühlung aus der Schmiedewärme (a) sowie temperaturabhängige Wärmeübergangskoeffizienten (HTC) der Spraykühlung zu 1.3505 im Vergleich zu HTC von Wasser zu 1.3505 (b) 
sich deutlich unterscheidenden HTC lässt sich direkt erkennen, dass die verschiedenen Kühlstrategien in unterschiedlichen Temperaturprofilen während der Abkühlphase resultieren. Bei der Abkühlung in Wasser variiert der HTC über den Temperaturbereich von 1000 bis $20^{\circ} \mathrm{C}$ erwartungsgemäß, da die verschiedenen Abkühlstufen Filmsieden, Blasensieden und Konvektion durchlaufen werden. Mit der Spraykühlung werden unterhalb einer Temperatur von etwa $300^{\circ} \mathrm{C}$ vergleichsweise höhere HTCs erzeugt. Durch die Zerstäubung des Wassers entstehen kleine Tropfen, welche eine große Oberfläche in Relation zu ihrem Volumen haben. In Folge der vergrößerten Kontaktfläche findet ein intensivierter Wärmeübergang statt. Oberhalb von $300{ }^{\circ} \mathrm{C}$ werden im Sprayfeld geringere HTCs als bei der Wasserabkühlung erzeugt. Bei diesen Temperaturen bildet sich entsprechend des Leidenfrosteffektes ein Dampffilm an der heißen Werkstückoberfläche, welcher den direkten Kontakt von Tropfen und Werkstück verhindert. Mit den eingestellten Luft- und Wasserdruckparametern reicht die kinetische Energie der Tropfen nicht aus, diesen Dampffilm zu durchdringen.

\subsection{Modellspezifische Anforderungen}

Wie in Abb. 3a dargestellt, lassen sich die bei der Warmumformung von Stählen auftretenden Phänomene auf thermische, metallurgische und mechanische Effekte zurückführen, deren Ausprägungen auf der chemischen Zusammensetzung der Legierung beruhen. Diese unterschiedlichen Effekte werden in der FE-Simulation thermomechanischer Prozesse häufig mittels additiver Verzerrungszerlegung berücksichtigt, z.B. in [15, 16] und [17]. Nach dieser Methode wird das gesamte Verzerrungsinkrement aus der Summe der Verzerrungen berechnet, die durch elastische,

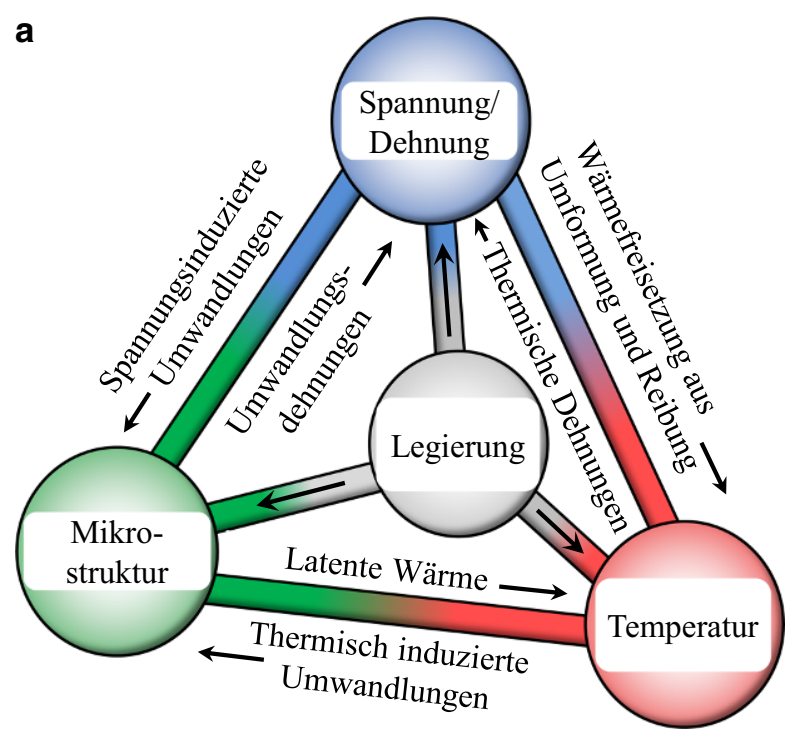

plastische, thermische, umwandlungsinduzierte und umwandlungsplastische Effekte verursacht werden (Abb. 3b). Vor diesem Hintergrund wird deutlich, dass insbesondere die simulative Prognose der aus Warmumformprozessen resultierenden Eigenspannungen eine Herausforderung darstellt, zumal die Spannungsverteilung im Bauteil durch eine inhomogene und sich wechselseitig beeinflussende Verteilung der einzelnen Verzerrungsskomponenten aus den fünf oben genannten physikalischen Phänomenen entstehen kann [18].

Weiterhin ist zu beachten, dass die Eigenspannungen, die durch den thermo-mechanisch-metallurgischen Prozess entstehen, maßgeblich durch die Phasenumwandlungen beeinflusst werden. Diese Umwandlungen finden auf atomarer Ebene statt, was zu Verzerrungen auf mikroskopischer Ebene und damit zu Eigenspannungen führt. Generell existieren drei Arten von Eigenspannungen [19], wobei sich Eigenspannungen erster Art auf das gesamte Bauteil beziehen und als makroskopisch definiert werden. Die Differenz zwischen der mittleren Spannung eines Korns und der makroskopischen Eigenspannung wird als Eigenspannung zweiter Art betrachtet. Die ortsabhängigen Abweichungen der Spannungen innerhalb des Korns von der Summe der Eigenspannungen erster und zweiter Art werden wiederum als Eigenspannungen dritter Art bezeichnet. Durch klassische Röntgendiffraktometrie sind makroskopische Eigenspannungen messbar, die Einflüsse des zweiten und dritten Typs auf die Stabilität der makroskopischen Eigenspannungen jedoch nicht. Für eine vollständige Analyse der resultierenden Eigenspannungen in einem Bauteil sind daher weitere numerische Analysen auf mikroskopischer Ebene erforderlich.

b

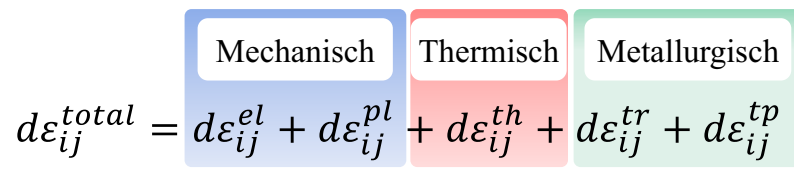

$d \varepsilon_{i j}^{e l}:$ Elastisches Verzerrungsinkrement

$d \varepsilon_{i j}^{p l}$ : Plastisches Verzerrungsinkrement

$d \varepsilon_{i j}^{t h}:$ Thermisches Verzerrungsinkrement

$d \varepsilon_{i j}^{t r}$ : Umwandlungsinduziertes Verzerrungsinkrement

$d \varepsilon_{i j}^{t p}:$ Umwandlungsplastisches Verzerrungsinkrement

Abb. 3 Wechselseitige thermomechanisch-metallurgische Werkstoffphänomene bei der Warmumformung (a) und Grundprinzipien der Methode der additiven Verzerrungszerlegung (b), [21] 
Dafür wurde eine Multilevel FEM, die sogenannte $\mathrm{FE}^{2}$ Methode, zur numerischen Analyse auf verschiedenen Skalen entwickelt [20]. Diese Simulationsmethode ermöglicht es durch das Konzept des Mikro-Makro-Übergangs, das effektive, makroskopische Verhalten von komplexen, mikroheterogenen Materialien durch die direkte Modellierung auf der unteren (Mikro-) Skala zu beschreiben. Dabei können neben den in diesem Beitrag berücksichtigten klassischen, mechanischen Größen, wie z.B. Verzerrungen und Spannungen, auch weitere physikalische Effekte, z. B. magnetische, modelliert werden. Den numerischen Auswertungspunkten in der FE-Diskretisierung des Bauteils auf der Makroskala wird ein repräsentatives Volumenelement (RVE) zugeordnet, welches aus der FE-Diskretisierung des heterogenen Materials auf der Mikroskala besteht. Mittels Lokalisierungs- und Homogenisierungstechniken werden die Wechselwirkungen zwischen den beiden Skalen ausgetauscht.

Auf der Mikroskala werden die entsprechenden Materialeigenschaften modelliert und das zugehörige Randwertproblem unter Verwendung der makroskopischen Belastungszustände gelöst. Anschließend werden daraus effektive Größen berechnet, um das makroskopische Randwertproblem aufzustellen und zu lösen.

\section{Einsatz mehrskaliger FE-Modellierung zur Prozessauslegung}

\subsection{Einskalige Betrachtung}

Der im Abschn. 2.1 beschriebene Demonstratorprozess inklusive seiner Randbedingungen wurde in simufact.forming v16 zunächst mit einem einskaligen makroskopischen FEModell nachgebildet. Der Modellaufbau wurde detailliert in [22] beschrieben. Eine schematische Darstellung des Modells wird in Abb. 4 gezeigt. Die Simulation startet zu Beginn des Umformprozesses mit einer thermisch expandierten Probengeometrie bei $1000^{\circ} \mathrm{C}$ und dem Ausgangszustand eines $100 \%$ austenitischen Gefüges. Die Werkzeuge werden als wärmeleitende starre Körper aus dem Werkstoff AISI Inconel 718 (DIN 2.4668) mit einer spezifischen Wärmekapazität von $435 \mathrm{Jkg}^{-1} \mathrm{~K}^{-1}$ und einer Wärmeleitfähigkeit von $11,4 \mathrm{Wm}^{-1} \mathrm{~K}^{-1}$ gemäß den Angaben des Materiallieferanten modelliert [23].

Zur Berechnung der Fließspannung wird das sogenannte GMT-Modell (Gesellschaft für Maschinentechnik mbH) eingesetzt, welches in [10] vorgestellt und kalibriert wurde. Darüber hinaus wird das Umformungs-Zeit-TemperaturUmwandlungsdiagramm (UZTU) aus [24] zur Modellierung des Gefügeumwandlungsverhaltens verwendet. Des Weiteren wird der umwandlungsplastische Effekt von Phasenumwandlungen, die unter überlagerten Spannungen auftreten, berücksichtigt. Die Umwandlungsplastizitätsparameter, wurden über eine experimentell-numerische Methode werkstoff- und phasenspezifisch in [22] ermittelt. Für eine ganzheitliche numerische Betrachtung der Prozesskette der Warmumformung ist es zudem notwendig, eine Vielzahl weiterer Werkstoffparameter phasenspezifisch in die Simulation einzubeziehen, um die Polymorphie des Stahls korrekt abzubilden. Da eine experimentelle Bestimmung vieler Werkstoffparameter für die reine Gefügephase schwierig ist [25], wird die thermodynamische Berechnungssoftware JMatPro [26] eingesetzt. Mit Hilfe dieser Software werden, basierend auf empirischen Gleichungen, anhand der chemischen Zusammensetzung der Legierung phasenspezifische und temperaturabhängige Werte für spezifische Wärmekapazität, Wärmeleitfähigkeit, Wärmeausdehnungskoeffizient, Dichte, E-Modul, Poissonzahl, latente Wärme und Härte berechnet. Die Daten wurden bereits in tabellarischer Form in [11] veröffentlicht. Außerdem sind die HTC Werte für die Abkühlung im Wasser beziehungsweise im Spray aus Abschn. 2.2 in die FE-Modelle implementiert. Auf die Oberflächen, welche bei Spraykühlungsszenarien nicht mit dem Spray benetzt werden, wird der HTC zwischen Luft und 1.3505 aus [11] appliziert.

\subsection{Mehrskalige Betrachtung}

Wie bereits in Abschn. 2.3 erläutert, ermöglicht eine $\mathrm{FE}^{2}$ Simulation die Analyse physikalischer Größen auf verschiedenen Skalen, hier insbesondere der Eigenspannungen in Folge der Abkühlung und der damit verbundenen Phasentransformation von Austenit zu Martensit. Unter Verwendung der mehrskaligen Modelle, [27] und [28], wird die Abkühlung innerhalb der Schnittebene auf halber Höhe des zylindrischen Bauteils simuliert, siehe Abb. 4, um die Entwicklung der Eigenspannungen sowohl auf Bauteilebene als auch Mikroskala zu untersuchen. Die ersten Schritte der Prozesskette, d.h. Aufheizen und Stauchen, werden nicht mit dem mehrskaligen Modell simuliert. Jedoch wird die Ausgangskonfiguration für diese $\mathrm{FE}^{2}$-Simulation durch die deformierte Bauteilgeometrie und Temperatur nach dem Stauchen definiert. Diese Daten liefert die Simulation des Stauchprozesses mit dem einskaligen Modell aus Abschn. 3.1. Da dieses Modell auch den Temperaturanstieg aufgrund plastischer Deformationen berücksichtigt, werden diese Temperaturänderungen folglich auch bei der Initialisierung des mehrskaligen Modelles berücksichtigt. Darüber hinaus wird dieser Zustand als spannungsfrei aufgrund der hohen Umformtemperaturen angenommen. In den entwickelten Modellen wird die Phasentransformation von Austenit zu Martensit unter Berücksichtigung der temperaturabhängigen Änderung des Fließverhaltens miteinbezogen. Das dazu gewählte Materialmodell beschreibt elasto-plastisches Materialverhalten, siehe [29], mit tempe- 


\section{Einskaliges FE-Modell}

Oberes Werkzeug

(starr, wärmeleitend)

3D-Halbmodell des

Werkstücks

(elastisch-viskoplastisch)

Unteres Werkzeug

(starr, wärmeleitend)

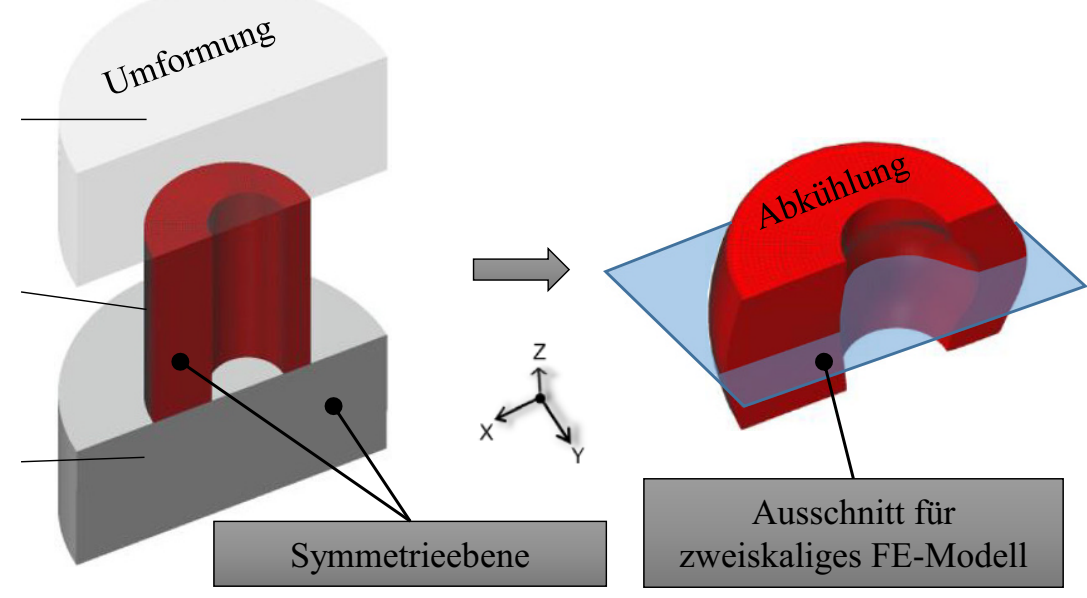

Zweiskaliges FE-Modell
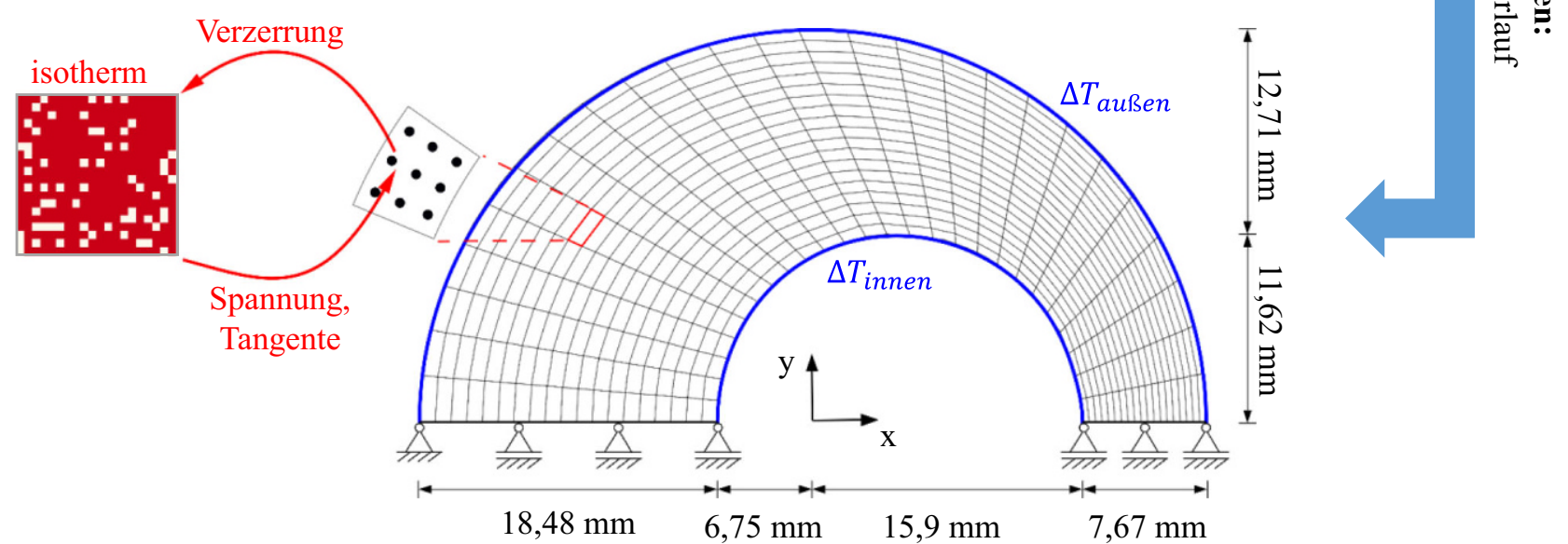

Abb. 4 Aufbau und Verknüpfung des mehrskaligen Simulationsmodells mit dem einskaligen Simulationsmodell inklusive der verwendeten Rahmenbedingungen [22]

raturabhängigen, phasenspezifischen Materialparametern, siehe [11], und integriert umwandlungsinduzierte Verzerrungen. Die Temperatur wird dabei aus der Lösung der Energiebilanz auf Makroskala gewonnen. Details zum Materialmodell sowie zur Wahl der phasenspezifischen und temperaturabhängigen Materialparameter finden sich in [27] und [28].

Für die zweiskalige Simulation des Schnittes durch den Zylinder wird ein ebener Verzerrungszustand auf beiden Skalen angenommen. Die Abmessung der Geometrie unter Ausnutzung der Symmetrie, ihre Diskretisierung sowie die Randbedingungen sind in Abb. 4 definiert. Aufgrund der Symmetrie kann der Wärmefluss in Umfangsrichtung über die Symmetrieachse ( $\mathrm{x}$-Achse) hinweg vernachlässigt werden. Der Temperaturverlauf auf Außen- und Innenrand aus der rein makroskopischen Simulation des Prozesses, welche in Abschn. 3.1 beschrieben wird, dient als Grundlage für die makroskopischen Temperaturrandbedingungen $\Delta T \mathrm{im}$ zweiskaligen Modell. Für diese wird jeweils für den Innenund Außenrand der gemittelte Temperaturverlauf auf den entsprechenden Rändern der Schnittfläche im einskaligen Modell aus Abschn. 3.1 berechnet.

Für das mikroskopische Randwertproblem wird eine künstliche, quadratische Mikrostruktur als RVE mit periodischen Randbedingungen gewählt, siehe Abb. 4 links unten. Auf dieser Skala wird die mikroskopische AustenitMartensit-Phasentransformation in der Materialmodellierung berücksichtigt, wie es in [27] und [28], detailliert vorgestellt ist.

Bei der numerischen Umsetzung der Sprayfeldabkühlung sind inhomogenere Temperaturverteilungen auf den Rändern in Bezug auf die Randbedingungen zu berück- 
Abb. 5 Zweiskaliges Randwertproblem für die Abkühlung im Sprayfeld

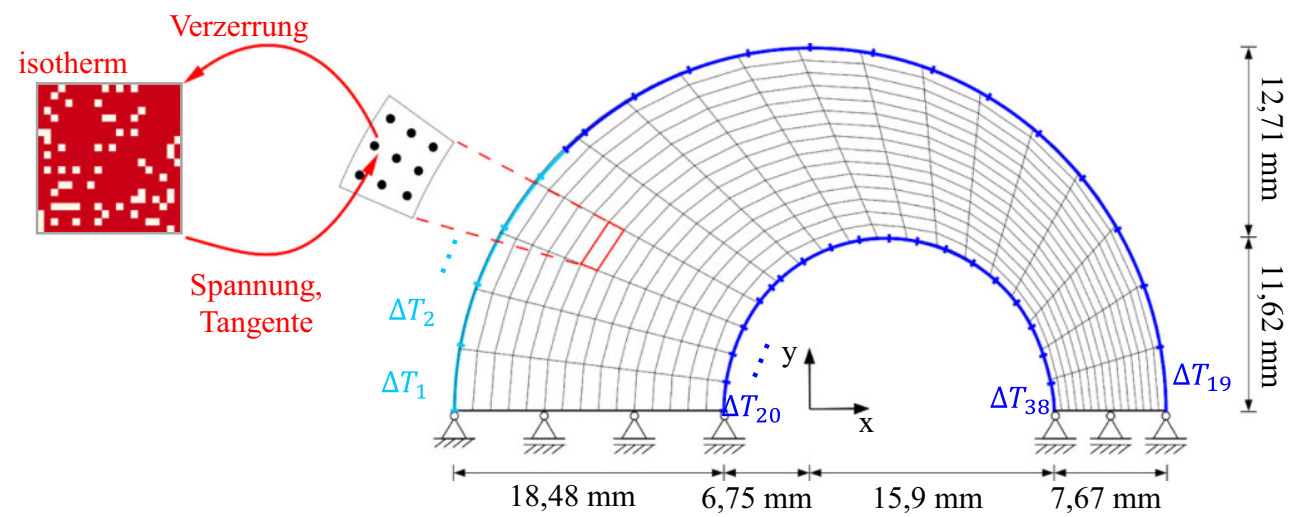

sichtigen. Der Rand wird hierzu in Teilabschnitte unterteilt, vgl. Abb. 5. Je Abschnitt wird die Temperatur des Knotens aus der entsprechenden einskaligen Simulation des Modells aus Abschn. 3.1 in der makroskopischen Diskretisierung aufgebracht, welcher betrachtet im Uhrzeigersinn am Teilabschnittsbeginn liegt.

\section{Numerische Prozessanalyse und experimentelle Evaluation der FE-Modelle}

\subsection{Numerische Prozessauslegung mittels makroskopischer trial-and-error FE- Simulationen}

Mit dem Ziel, einen geeigneten Prozessablauf zur Einstellung von Druckeigenspannungen in der Probenoberfläche zu finden, wurden mehrere Prozessvarianten mit dem makroskopischen FE-Modell simuliert. Die Untersuchungen in dieser Arbeit beschränken sich dabei auf kontinuierliche Abkühlvorgänge, welche unmittelbar nach dem Umformen starten und bei Erreichen der Raumtemperatur enden. Außerdem wird nur die in Abschn. 2.1 beschriebene Einstellung hinsichtlich Luft- und Wasserdruck mit den dort gezeigten HTC Werten betrachtet. Unter diesen Voraussetzungen kommen verschiedene Abkühlszenarien in Frage. Für vier Szenarien ist die simulative Prognose in Abb. 6 exemplarisch dargestellt: Die Abkühlung mit sechs Düsen, „Spray ganz“; die Abkühlung mit einer Düse, welche auf die dünnwandige Seite fokussiert ist, „Spray dünn“; die Abkühlung mit zwei Düsen, welche auf die Seiten der Probe gerichtet sind, „Spray seitlich“; sowie die Abkühlung mit einer Düse, welche auf die dickwandige Seite gerichtet ist, „Spray dick“. Die resultierenden tangentialen Eigenspannungen auf dem Umfang der Probe sind ebenfalls in Abb. 6 in Abhängigkeit vom Positionswinkel $\theta$ dargestellt. Vergleichend dazu ist die Verteilung der Eigenspannungen nach der Abkühlung in Wasser eingezeichnet. Diese Variante weist die höchsten Zugspannungen auf dem Probenumfang auf.
Mit den Varianten „Spray dünn“ und „Spray ganz“ werden ebenfalls hauptsächlich Zugeigenspannungen erzeugt. In den Prozessen mit den Varianten „Spray seitlich“ und „Spray dick“ treten dagegen Zug- und Druckspannungen auf.

Für die experimentelle Realisierung in Abschn. 4.2 sowie die tiefergehende mehrskalige numerische Prozessanalyse in Abschn. 4.3 wird die Prozessvariante „Spray dick“ gewählt, da hier die betragsmäßig höchsten Druckspannungen auftreten.

\subsection{Experimentelle Realisierung der numerisch ausgelegten Prozessstrategie}

Nach der experimentellen Herstellung der Proben mit der Prozessvariante „Spray dick“, werden die oberflächennahen Eigenspannungen auf dem Umfang der Probe in Abhängigkeit vom Positionswinkel $\theta$ mittels Röntgendiffraktometrie gemessen. Hierbei wird die gleiche Vorgehensweise wie bereits in [11] zur Messung der Eigenspannungen an den Proben, welche in Wasser abgekühlt wurden, angewendet. Ein Vergleich der Eigenspannungsergebnisse aus Experimenten und Simulationen in den Proben aus den Prozessvarianten „Wasser“ und „Spray dick“ ist in Abb. 7a dargestellt. Auf der dickwandigen Seite, ausgehend vom Winkel $\theta=0^{\circ}$ bei MP1 bis zu $\theta=45^{\circ}$, wurden mit der Variante „Spray dick“, wie mit Hilfe der Simulationen vorhergesagt, experimentell Druckspannungen von $\sigma_{\mathrm{t}}=-407 \mathrm{MPa}$ bzw. $\sigma_{\mathrm{t}}=-223 \mathrm{MPa}$ erzeugt. Damit sind die Eigenspannungen an diesen Positionen deutlich modifiziert, verglichen mit den Zugeigenspannungen von $\sigma_{\mathrm{t}}=216 \mathrm{MPa}$ für $\theta=0^{\circ}$ und $\sigma_{\mathrm{t}}=146 \mathrm{MPa}$ für $\theta=45^{\circ}$, die durch die einfache $\mathrm{Ab}$ kühlung im Wasser entstehen. $\mathrm{Ab} \theta=90^{\circ}$ erzeugen beide Strategien Zugeigenspannungen. Wie in den numerischen Analysen vorhergesagt, zeigen die experimentellen Ergebnisse also, dass die Prozessvariante „Spray dick“ für die Erzeugung von Druckeigenspannungen an den Positionen $\theta=0^{\circ}$ bis $\theta=45^{\circ}$ geeignet ist. Für eine weitere Anpassung der oberflächennahen Eigenspannungen könnte nicht nur 
Abb. 6 Numerische Studie über die Ausbildung der tangentialen Eigenspannungen auf dem Probenumfang in Folge möglicher Prozessszenarien mit Spraykühlung im Vergleich zur einfachen Abkühlung im Wasser
Messpunkte (MP) auf dem Umfang der Probe in Abhängigkeit des Winkels $\theta$
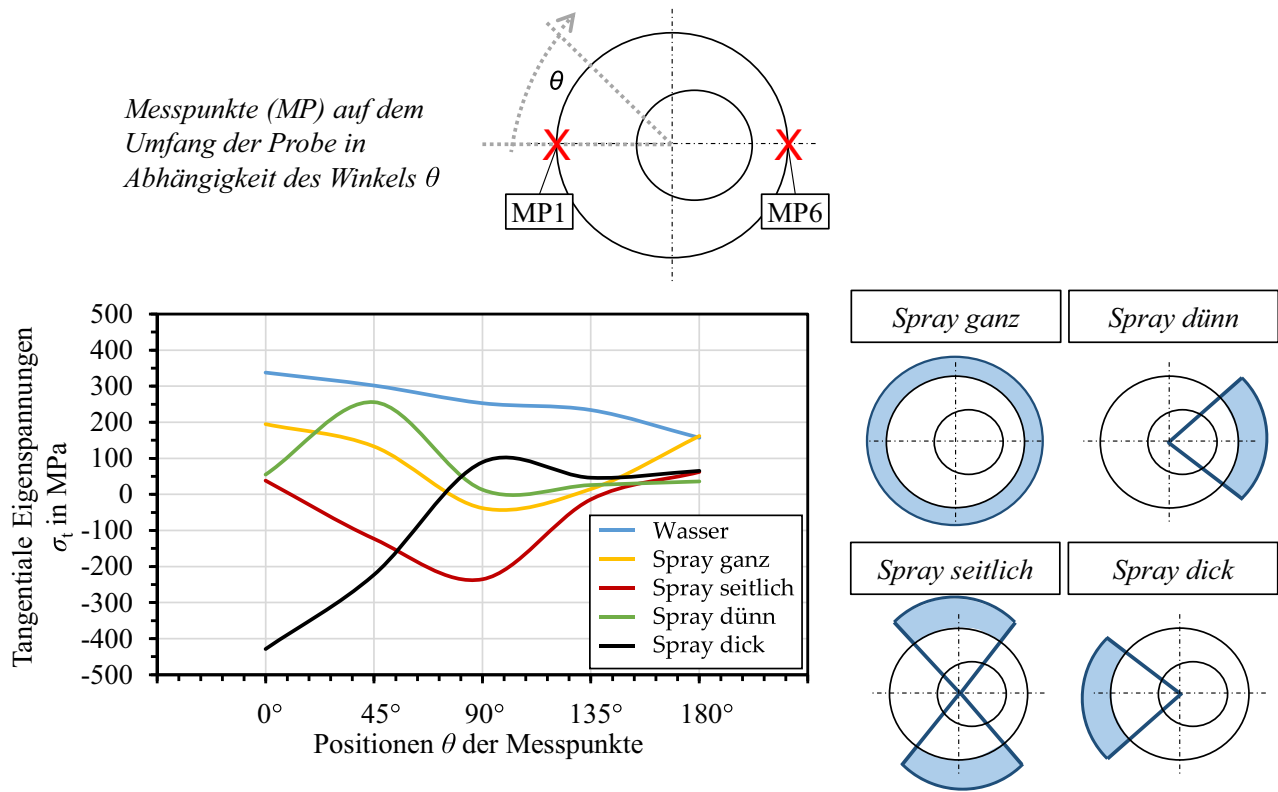

eine örtlich, sondern auch zeitlich angepasste Abkühlung mittels Sprayfeld numerisch und experimentell erprobt werden. Des Weiteren besteht die Möglichkeit, die Intensität der Spraykühlung und damit die Entwicklung der Eigenspannungen durch Variation von Luft- und Wasserdruck anzupassen.

Auf der dünnwandigen Seite zeigen die experimentellen Ergebnisse der Variante „Spray dick“ höhere Zugspannungen im Vergleich zur Simulation. Zum Beispiel werden bei MP6 $\sigma_{\mathrm{t}}=222 \mathrm{MPa}$ gemessen, im Gegensatz zur berechneten Spannung von $\sigma_{\mathrm{t}}=65 \mathrm{MPa}$. Dies kann darauf zurückzuführen sein, dass die Einflüsse aus metallurgischen Effekten wie die Versetzungsdichte aufgrund der Austenitumwandlung, lokale Gradienten der Fließspannung an den Phasengrenzen oder die erzwungene Auflösung von Kohlenstoffatomen im martensitischen Gitter nur bei konstanten Randbedingungen in der Werkstoffcharakterisierung berücksichtigt werden. So werden z.B. die UZTU-Diagramme nach der deutschen Norm [30] für ein Gefüge nach einer zehnminütigen Haltezeit bei Austenitisierungstemperatur und direktem Abschrecken ermittelt. Gleiches gilt für die Bestimmung der Umwandlungsplastizitätskonstanten, die durch einen experimentell-numerischen Ansatz für eine kontinuierliche Abkühlung von der Austenitisierungstemperatur bestimmt werden. Dies erklärt eine höhere Abweichung zwischen experimentellen und numerischen Ergebnissen, insbesondere an den Stellen, die eine verzögerte Abkühlung erfahren, wie es an der dünnwandigen Probenseite der Fall ist.

Um eine Beeinflussung der Modifikationen der Eigenspannungen in den Proben aus der Variante „Spray dick“ gegenüber der Variante „Wasser“ von der Gefügezusammensetzung oder einer veränderten Geometrie auszuschlie- ßen, wurden metallografische Untersuchungen und optische Geometrieanalysen vorgenommen. Die Vorgehensweise hierfür wurde bereits in [11] veröffentlicht. Für die Proben aus beiden Strategien lässt sich aus den Härtewerten von ca. $800 \mathrm{HV}$ im gesamten Probenquerschnitt (Abb. 7b) und den lichtmikroskopischen Aufnahmen (Abb. 7d) schließen, dass es sich um vollständig gehärtete martensitische Proben handelt. Abb. 7c zeigt die optisch vermessene Geometrie einer Probe, welche in Wasser abgekühlt wurde. Der Konturplot der Abweichung $e$ auf dieser Fläche gibt den dreidimensionalen Abstand in senkrechter Richtung zur Oberfläche der Geometrie einer Probe, welche mit der Prozessvariante „Spray dick“ hergestellt wurde, an. Die Abweichung von $0 \mathrm{~mm}$ zeigt dabei eine perfekte Übereinstimmung zwischen den Geometrien aus beiden Strategien. Das Vorzeichen des Abweichungswertes gibt an, ob der entsprechende Bereich der Geometrie aus der Variante „Spray dick" außerhalb (positiv) oder innerhalb (negativ) der Werkstückform aus Variante „Wasser“ liegt. Die unterschiedlichen thermomechanischen Strategien führen zu lokalen Abweichungen von weniger als $0,5 \mathrm{~mm}$. Obwohl die Proben auf unterschiedliche Weisen hergestellt wurden, besitzen sie trotzdem nahezu gleiche Geometrie- und Gefügeeigenschaften. An den Proben aus dem Prozess „Spray dick“ wurden demnach nur die Eigenspannungen gegenüber den Proben aus der Variante „Wasser“ verändert. Daraus lässt sich schließen, dass eine Modifikation der Eigenspannungen durch geschickte Prozessführung möglich ist. 
a

Messpunkte (MP) auf dem

Umfang der Probe in

Abhängigkeit des Winkels $\theta$
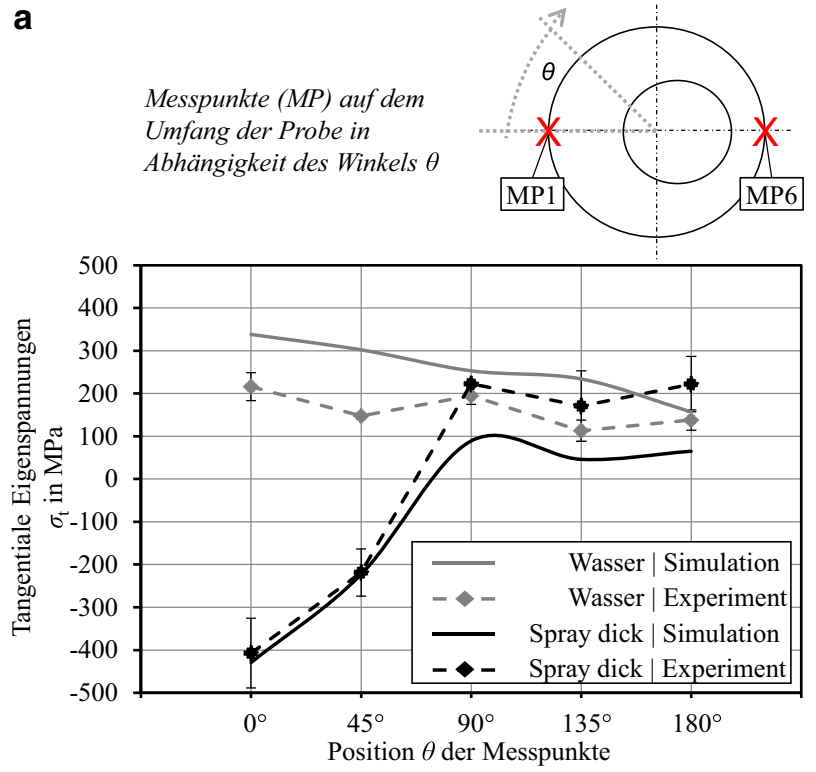

C

Geometrische Abweichung e in mm der Proben aus Wasser- und Spraykühlung

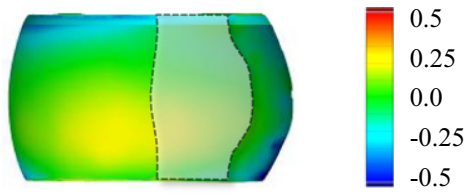

b

Messpunkte (MP) auf mittlerer Höhe der Probe
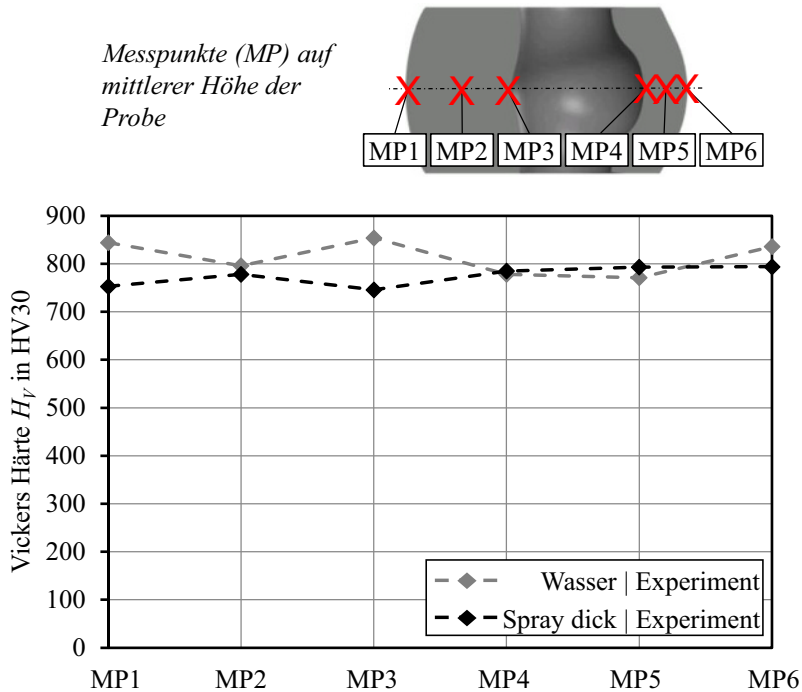

d
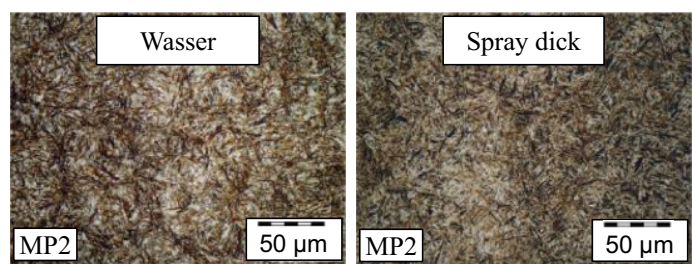

Abb. 7 Vergleich der nach Varianten „Wasser“ sowie „Spray dick“ hergestellten Proben im Hinblick auf Eigenspannungen aus Experiment und Simulation (a), Vickers Härte (b), Geometrieabweichung (c) und Gefüge (d)

\subsection{Numerische Prozessanalyse mit einskaligen und mehrskaligen FE-Modellen}

In den vorangegangenen Abschnitten wurde eine hinsichtlich der finalen, makroskopischen Eigenspannungsverteilung verbesserte Prozessvariante „Spray dick“ numerisch ermittelt und die Ergebnisse experimentell abgesichert. Um nun die Effekte, die zu dieser veränderten Spannungsverteilung führen und die Einflüsse der mikroskopischen Eigenspannungen zu analysieren, werden die FE-Modelle aus Kap. 3 verwendet. Diesen Ergebnissen werden die Resultate aus der ursprünglichen Prozessvariante „Wasser“ gegenübergestellt.

Als primäre Prozessgröße wird zunächst der Temperaturverlauf während des Abkühlvorgangs im Experiment und der makroskopischen Simulation an den Messpunkten MP 1 und MP 6 verglichen, um die aufgebrachten Temperaturrandbedingungen zu validieren. In Abb. 8 sind diese für die beiden Prozessvarianten gegenübergestellt und zeigen sehr gute Übereinstimmungen. Sichtbar wird auch der Einfluss des einseitig aufgebrachten Sprayfelds, woraus ein deutlich verlangsamter Abkühlvorgang am Messpunkt MP6 resultiert. Aufgrund der guten Übereinstimmung können die aus der makroskopischen Simulation extrahierten Temperaturen auf den Außenrändern als Temperaturrandbedingungen auf die makroskopische Diskretisierung im Mehrskalenmodell angewendet werden.

In Abb. 9 wird die Entwicklung der Tangentialspannungen in den Messpunkten MP 1 und MP 6 über der Abkühlzeit dargestellt, welche sich aus den Simulationen mit dem einskaligen und dem mehrskaligen Modell für die Abkühlvarianten „Wasser“ und „Spray dick“ ergeben. Da im Abschn. 4.2 bereits die gute Übereinstimmung der makroskopischen Simulation mit den Experimenten im finalen $\mathrm{Zu}-$ stand, sprich nach der Abkühlung, dargestellt wurde, werden die zeitlichen Spannungsverläufe dieser Simulation im Folgenden als Referenz für die weitere Analyse betrachtet.

Nach der Wasserabkühlung zeigen sich die bereits zuvor beobachteten Zugeigenspannungen, siehe Abb. 9a. Diese lassen sich durch die entstehenden Temperaturgradienten zwischen der Probenoberfläche und dem Probenkern sowie den dadurch verursachten thermischen und umwandlungsinduzierten Verzerrungseffekten erklären. Die Zugspannungen stellen sich zu Beginn der Abkühlung aufgrund der thermischen Kontraktion des Materials an der Probenoberfläche ein, die gegen den Widerstand des noch thermisch expandierten Probenkerns wirkt. Bei Erreichen der Martensitstarttemperatur an der Probenoberfläche verursacht die Volumenexpansion in Folge der Martensitumwandlung ab ca. 5,6s Abkühlzeit eine Umkehr des Spannungsvorzei- 
a

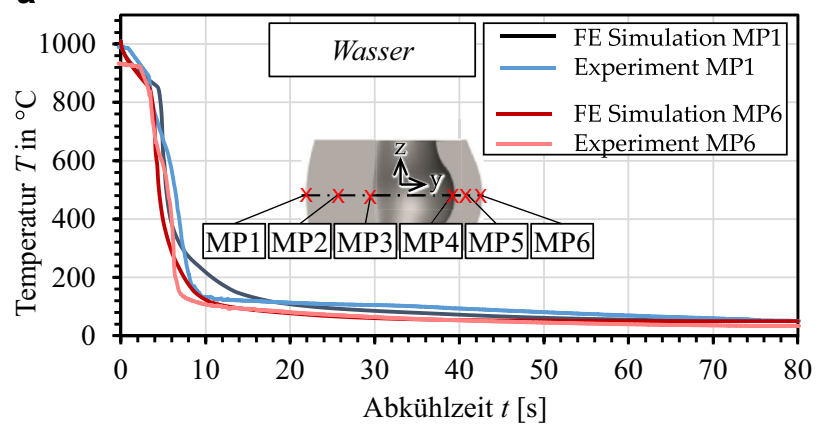

b

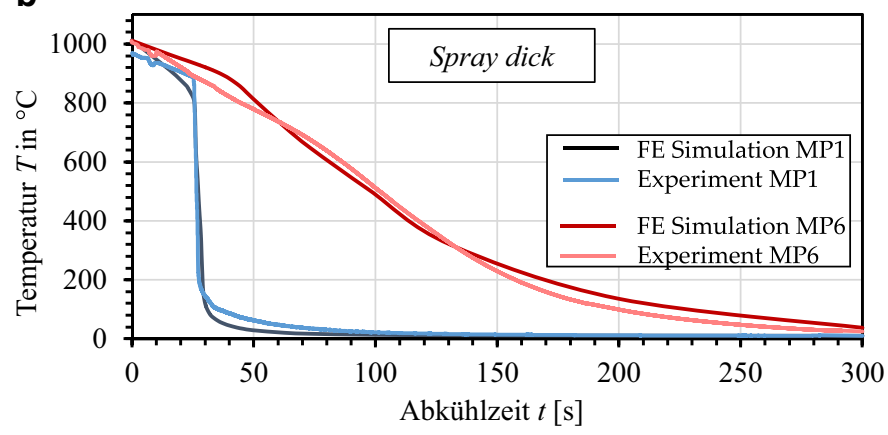

Abb. 8 Abgleich der Temperaturrandbedingungen von experimentellen und numerischen Untersuchungen für die Variante „Wasser“ (a) und die Variante ,Spray dick“(b)
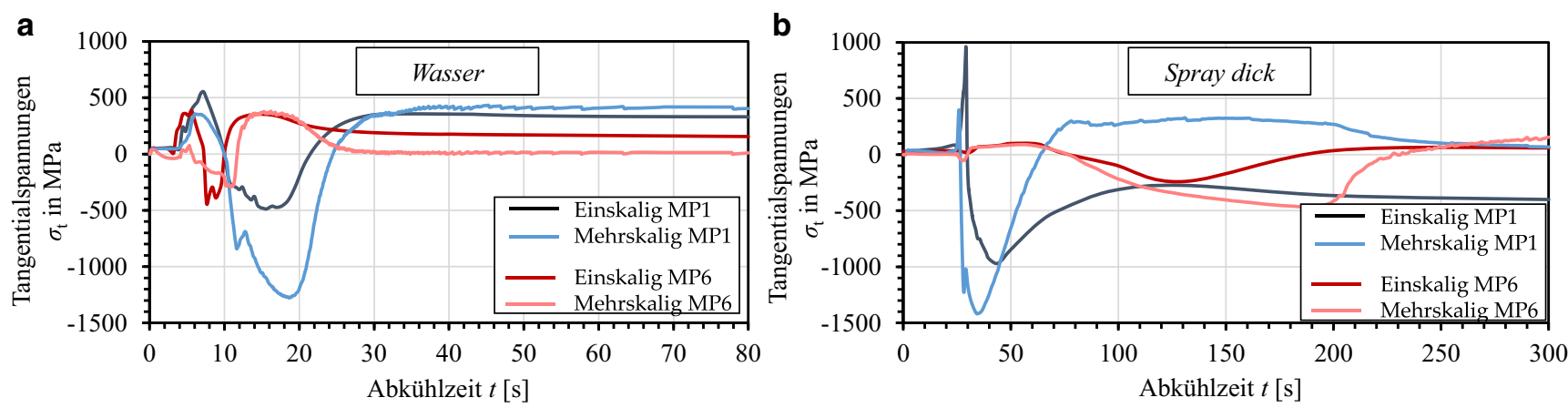

Abb. 9 Verlauf der Tangentialspannungen während der Abkühlung für die Prozessvariante „Wasser“ (a) und „Spray dick“ (b) aus den einskaligen und mehrskaligen Simulationen

chens. Die weitere Abkühlung verursacht auch im Probenkern die Volumenexpansion entgegen dem Widerstand der bereits erhärteten Randbereiche und Zugeigenspannungen an der Probenoberfläche entstehen.

Die resultierenden Spannungsverläufe der zweiskaligen Simulation des Prozesses der Wasserabkühlung zeigen qualitative Übereinstimmung verglichen mit der einskaligen Simulation. Die quantitativen Unterschiede sind wahrscheinlich auf die Vernachlässigung des Wärmeabflusses senkrecht zur Schnittebene, die gemittelten Temperaturrandbedingungen und die fehlende Berücksichtigung von latenter Wärmeentwicklung und umwandlungsplastischen Effekten in der mikroskopischen Materialmodellierung zurückzuführen.

Bei der Abkühlung „Spray dick“ laufen die thermischen und umwandlungsinduzierten Effekte anders ab. Mit beginnender Abkühlung durch das Spray kontrahiert sich das Material an der Oberfläche der dickwandigen Probenseite (MP 1), was zu Zugspannungen bei beiden numerischen Modellen führt, vgl. Abb. 9b. Nach hinreichender Abkühlung, findet in den oberflächennahen Bereichen um MP1 die martensitische Gefügeumwandlung statt, welche mit der Volumenexpansion sowie dem örtlichen Anstieg der Dehngrenze des Materials einhergeht. Zu diesem Zeitpunkt liegen dort bereits hohe Druckspannungen vor, welche eben- falls von beiden numerischen Modellen, wenn auch mit leichtem Zeitversatz, abgebildet werden. In der einskaligen Simulation schwächen sich diese Druckspannungen ab, sobald sich die martensitische Umwandlungsfront anschlieBend in Richtung des Probenkerns fortsetzt und auch hier die Volumenexpansion stattfindet. Die zeitlich verzögerte thermische Kontraktion im Inneren des Materials sowie die auf dem Umfang der Probe fortlaufende Volumenexpansion aufgrund der Martensitumwandlung sorgen jedoch für die Aufrechterhaltung des Druckspannungszustandes an der dickwandigen Seite. In der mehrskaligen Simulation findet hingegen ein erneuter Umschlag der Tangentialspannung in den positiven Wertebereich statt. Der Grund für diesen Unterschied liegt in der Ausgangskonfiguration für das mehrskalige Modell begründet. Dieser wird wie in Abschn. 2.3 beschrieben als spannungs- und verzerrungsfrei angenommen, wobei in Folge des Stauchens vor dem Beginn des Abkühlens plastische Verzerrungen vorliegen, was zur lokalen Materialverfestigung führt. Daraus resultiert ein später einsetzendes Fließen des Materials und somit eine andere Spannungsentwicklung. Der weitere Verlauf ähnelt dem der rein makroskopischen Simulation mit konstantem Versatz. Um eine Annäherung an die Ergebnisse der Experimente bzw. der einskaligen Simulation zu erreichen, sollen die 

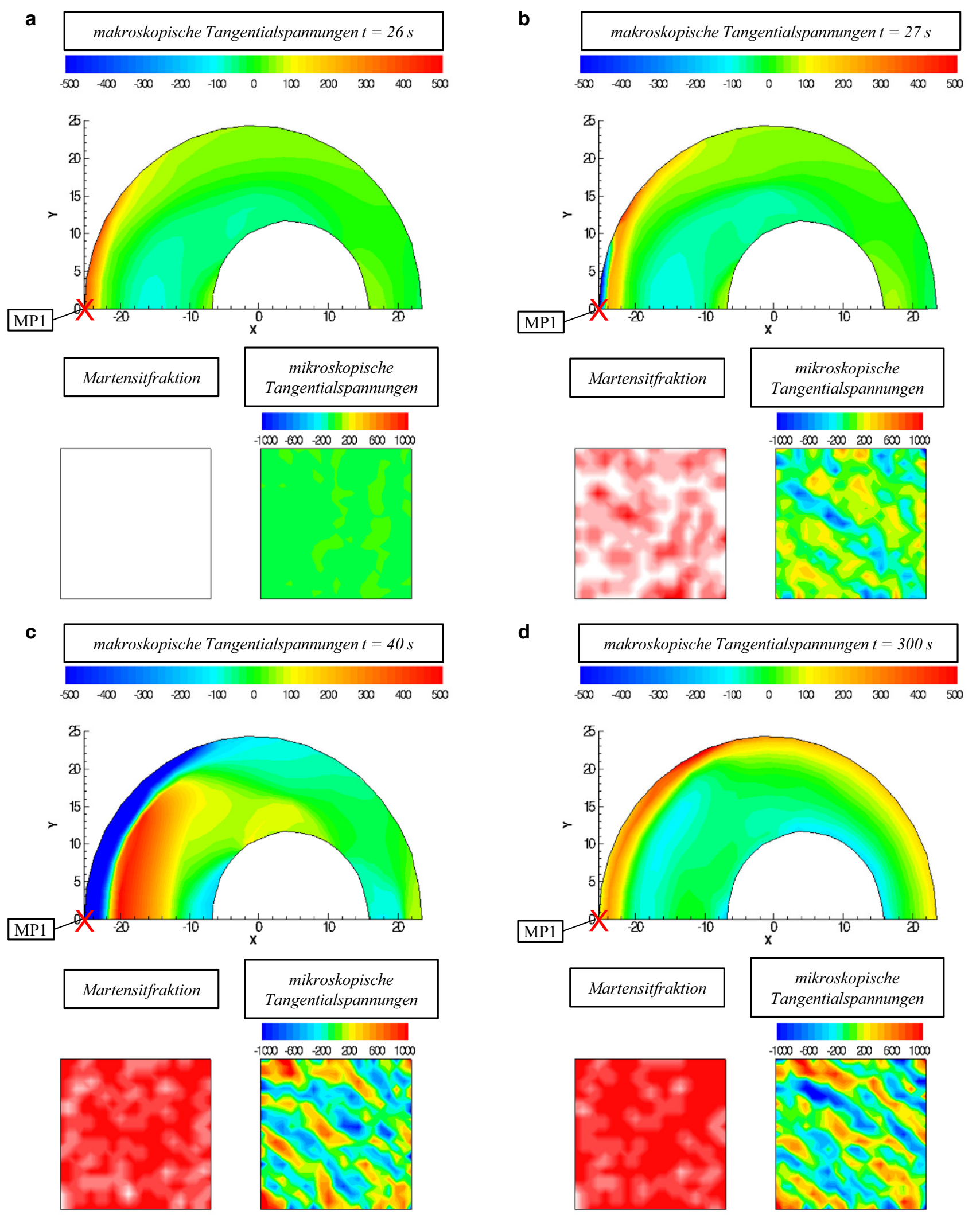

Abb. 10 Makroskopische Tangentialspannungen im Bauteilschnitt sowie die Martensitfraktion (rot) und die mikroskopischen Tangentialspannungen im RVE an MP1 für charakteristische Zeitpunkte $t=26 \mathrm{~s}(\mathbf{a}), \mathrm{t}=27 \mathrm{~s}(\mathbf{b}), \mathrm{t}=40 \mathrm{~s}(\mathbf{c})$ und $\mathrm{t}=300 \mathrm{~s}(\mathbf{d})$ 
genannten, vernachlässigten Effekte in der Materialmodellierung zukünftig berücksichtigt werden.

Zuletzt findet die Martensitumwandlung an der dünnwandigen Seite statt. Zu diesem Zeitpunkt liegen an MP6 Druckspannungen durch die vorangegangene Volumenausdehnung infolge der Martensitumwandlung in den Nachbarbereichen vor. Die Phasenumwandlung an MP6 findet also unter überlagerten Druckspannungen statt, die entsprechend des umwandlungsplastischen Effektes die Volumenausdehnung in Umfangsrichtung deutlich abschwächen. AbschlieBend führt die thermische Kontraktion am MP6 gegen den Widerstand der bereits erkalteten umliegenden Materialbereiche zu Zugeigenspannungen.

Trotz der bestehenden Diskrepanz in den finalen Eigenspannungswerten, werden im Folgenden die Möglichkeiten dargestellt, die das mehrskalige Modell hinsichtlich der Untersuchung verschiedener physikalischer Größen auf der Mikroskala bietet. Durch die Analyse mikroskopischer Größen kann ein Rückschluss über den Einfluss der Mikrostrukturevolution und die Auswirkungen auf den Eigenspannungszustand möglich gemacht werden. Dies ist besonders dann von Interesse, wenn ein repräsentatives Volumenelement auf der Grundlage einer realen Mikrostruktur verwendet wird. $\mathrm{Zu}$ diesen Größen zählen beispielsweise die Verteilung der Tangentialspannungen und die Martensitfraktion. Für charakteristische Zustände während der Sprayabkühlung sind in Abb. 10 neben der makroskopischen Tangentialspannungsentwicklung, das mikroskopische Pendant und die Martensitevolution an MP1 dargestellt. So zeigt Abb. 10a die Zugspannungen aufgrund der thermischen Kontraktion vor Einsetzen der Martensitumwandlung und Abb. 10b die Druckspannungen mit Einsetzen der Martensitumwandlung. Im weiteren Verlauf, siehe Abb. 10c, wird eine Druckspannungsspitze erreicht, welche in der mehrskaligen Simulation mit Einsetzen der Martensitumwandlung im Kern des Bauteils jedoch in Zugspannungen umschlägt, Abb. 10d. Per Definition berechnen sich makroskopische Spannungen aus dem Volumenmittel der mikroskopischen Spannungen. Als wesentliches Fazit zeigt sich hier, dass sich keine konstante mikroskopische Zugeigenspannungsverteilung einstellt, sondern lokale positive und negative Spannungsspitzen entstehen. Diese kann man gröBeren Martensit- bzw. Austenitbereichen zuordnen. Mittels weitergehenden Analysen, die diese Beziehung tiefer untersuchen, ist es möglich, ein physikalisch korrektes Verständnis der mikroskopischen Wechselwirkungen zu erlangen. Hieraus können in einem späteren Schritt der Einfluss von Eigenspannungen auf lokale Ermüdungs- oder Versagensvorgänge untersucht werden. Auch die Korrelation zwischen der Mikrostrukturevolution und sich einstellender Spannungsspitzen kann hiermit tiefer analysiert werden. Diese Rückschlüsse können dazu genutzt werden, die Materialmodellierung für das einskalige Simulationsmodell zu erweitern und/oder zu verbessern, um beispielsweise die Abweichung der Eigenspannungsberechnung an der dünnwandigen Seite gegenüber den experimentellen Ergebnissen, beschrieben in Abschn. 4.2, zu reduzieren.

\section{Zusammenfassung und Ausblick}

In dieser Arbeit wird mit Hilfe von FE-Simulationen die Variante eines Warmumformprozesses identifiziert, bei der sich mittels angepasster Spraykühlung aus der Umformwärme Druckeigenspannungen an der Oberfläche der Proben aus 1.3505 erzeugen lassen. Die Prognose der resultierenden Eigenspannungen kann mittels experimenteller Untersuchungen bestätigt werden. Darüber hinaus lässt sich mittels metallografischer Analysen sowie optischer Geometrievermessungen nachweisen, dass die Proben mit modifizierten Eigenspannungen die gleichen Geometrie- und Gefügeeigenschaften aufweisen, wie die Proben aus einem Prozess mit einfacher Abkühlung im Wasser. Somit wird gezeigt, dass die Modifizierung der Eigenspannungen allein durch geschickte Prozessführung möglich ist.

Im Zuge der Evaluation des makroskopischen FE-Modells wurde festgestellt, dass die Eigenspannungsprognosen in den Bereichen der Probe, welche eine verzögerte Abkühlung erfahren, eine geringere Genauigkeit aufweisen, als an Stellen, welche direkt abgeschreckt werden. Eine anwendungsorientierte Materialcharakterisierung könnte hier zur Verbesserung der Prognosegenauigkeit beitragen. Das verwendete mehrskalige Simulationsmodell weist noch Schwächen bei der Abbildung der Sprayfeldkühlung auf, welche auf vernachlässigte Effekte, wie z.B. umwandlungsplastische Verzerrungen, zurückzuführen sind und in den nächsten Modellerweiterungen berücksichtigten werden sollen. Jedoch werden bereits hier die Potenziale einer mehrskaligen Modellierung hinsichtlich der mikroskopischen Spannungsentwicklung vorgestellt, welche Aussagen über die Eigenspannungen erster und dritter Art liefern können.

In zukünftigen Untersuchungen könnte eine weitere Modifikation des Eigenspannungsprofils erfolgen, mit dem Ziel, Druckspannungen über den gesamten Umfang der Probe zu erzielen. Hierfür kann eine nicht nur örtlich, sondern auch zeitlich angepasste Abkühlung mittels Sprayfeld numerisch und experimentell erprobt werden. Des Weiteren besteht die Möglichkeit die Intensität der Spraykühlung durch Variation von Luft- und Wasserdruck anzupassen. Auch ist eine Anwendung der eingesetzten Methodik auf andere Schmiedestähle interessant, um die industrielle Tauglichkeit der Vorgehensweise zu prüfen. Abschließend sollte das Verfahren zur Optimierung der Eigenschaften eines komplexen Bauteils aus dem industriellen Umfeld angewendet werden. Auf diese Weise lässt sich das Po- 
tenzial der umformtechnisch induzierten Eigenspannungen unter realen Betriebsbedingungen erproben.

Danksagung Die Autoren bedanken sich für die vom Center for Computational Sciences and Simulation (CCSS) der Universität DuisburgEssen zur Verfügung gestellte Rechenzeit auf dem Supercomputer magnitUDE (DFG-Förderung INST 20876/209-1 FUGG, INST 20876/243-1 FUGG) am Zentrum für Informations- und Mediendienste (ZIM).

Förderung Gefördert durch die Deutsche Forschungsgemeinschaft (DFG) - 374871564 (BE 1691/223-2; SCHR 570/33-2; BR 5278/3-2) im Rahmen des Schwerpunktprogramms SPP 2013.

Funding Open Access funding enabled and organized by Projekt DEAL.

Open Access Dieser Artikel wird unter der Creative Commons Namensnennung 4.0 International Lizenz veröffentlicht, welche die Nutzung, Vervielfältigung, Bearbeitung, Verbreitung und Wiedergabe in jeglichem Medium und Format erlaubt, sofern Sie den/die ursprünglichen Autor(en) und die Quelle ordnungsgemäß nennen, einen Link zur Creative Commons Lizenz beifügen und angeben, ob Änderungen vorgenommen wurden.

Die in diesem Artikel enthaltenen Bilder und sonstiges Drittmaterial unterliegen ebenfalls der genannten Creative Commons Lizenz, sofern sich aus der Abbildungslegende nichts anderes ergibt. Sofern das betreffende Material nicht unter der genannten Creative Commons Lizenz steht und die betreffende Handlung nicht nach gesetzlichen Vorschriften erlaubt ist, ist für die oben aufgeführten Weiterverwendungen des Materials die Einwilligung des jeweiligen Rechteinhabers einzuholen.

Weitere Details zur Lizenz entnehmen Sie bitte der Lizenzinformation auf http://creativecommons.org/licenses/by/4.0/deed.de.

\section{Literatur}

1. Löhe D, Lang K-H, Vöhringer O (2002) Residual stresses and fatigue behavior. In: Totten, Howes M, Inoue T (Hrsg) Handbook of residual stresses and deformation. ASM International, Novelty

2. Withers PJ (2007) Residual stress and its role in failure. Rep Prog Phys (70):2211-2264, https://doi.org/10.1088/0034-4885/70/12/ R04

3. Löhe D, Hoffmann JE (2002) Influence of macro residual stresses on the fatigue behavior smooth and notched specimens made from a high strength steel. J Mater Manuf (111):704-710, https://doi.org/ 10.4271/2002-01-1408

4. Pape F, Neubauer T, Maiß O, Denkena B, Poll G (2017) Influence of residual stresses introduced by manufacturing process on bearing endurance time. Tribol Lett 65(70), https://doi.org/10.1007/ s11249-017-0855-3

5. Kritzler J, Wübbenhorst W (2002) Inducing compressive stresses through controlled shot peening. In: Totten, Howes M, Inoue T (Hrsg) Handbook of residual stress and deformation. ASM International, Novelty

6. Grum J (2002) Induction hardening. In: Totten, Howes M, Inoue T (Hrsg) Handbook of residual stress and deformation. ASM International, Novelty

7. Totten G, Howes M, Inoue T (2002) Handbook of residual stress and deformation of steel. ASM International,

8. Bach F-W, Kerber K (2014) Prozesskette Präzisionsschmieden. Springer Vieweg, Berlin, Heidelberg
9. Gegel HL, Malas JC (1993) Computer-aided process design for bulk forming. In: Semiatin SL (Hrsg) Handbook of forming and forging, Bd. 14. ASM International, Ohio

10. Behrens B-A, Schröder J, Wester H, Brands D, Uebing S, Kock C (2021) Experimental and numerical investigations on the development and stability of residual stresses arising from hot forming processes. 13th International Conference on Technology of Plasticity (ICTP),

11. Behrens B-A, Schröder J, Brands D, Scheunemann L, Niekamp R, Chugreev A, Sarhil M, Uebing S, Kock C (2019) Experimental and numerical investigations on the development of residual stresses in thermo-mechanically processed Cr-alloyed steel 1.3505. Metals 9(4), https://doi.org/10.3390/met9040480

12. Kaymak Y (2007) Simulation of metal quenching processes for the minimization of distortion and stresses. Otto-von-Guericke-Universität Magdeburg, Magdeburg

13. Nürnberger F, Diekamp M, Moritz J, Wolf L, Hübner S, Behrens B-A (2014) Spray cooling of early extracted hot stamped parts. TMS 2014: 143rd Annual Meeting \& Exhibition, S 983-990

14. Behrens B-A, Maier H, Neumann A, Moritz J, Hübner S, Gretzki T, Nürnberger F, Spiekermeier A (2015) Press hardening of tubes with additional interior spray cooling. Int $\mathrm{J}$ Mech Mechatron Eng (9):957-963, https://doi.org/10.15488/4178

15. Denis S, Gautier E, Simon A, Beck G (1985) Stress-phase-transformation interactions-basic principles, modelling and calculation of internal stresses. Mater Sci Technol, https://doi.org/10.1179/mst. 1985.1.10.805

16. Simsir C (2008) 3D finite element simulation of steel quenching in order to determine the microstructure and residual stresses. Middle East Technical University,

17. Behrens B-A, Schrödter J (2014) Numerical simulation of phase transformation during the hot stamping process. In: Proceedings of the 5th international conference on thermal process modeling and computer simulation, Bd. 17-18, S 179-190

18. Behrens B-A, Olle P, Schäffner C (2008) Process simulation of hot stamping in consideration of transformation-induced stresses. In: Numisheet, S 557-562

19. Macherauch E, Wohlfahrt H, Wolfstied U (1973) Zur zweckmäßigen Definition von Eigenspannungen. Härterei Tech Mitt Z Werkstoffe Wärmebehandl Fert 3(28):201-211

20. Schröder J (2014) A numerical two-scale homogenization scheme: the FE $^{2}$-method. In: Schröder J, Hackl K (Hrsg) Plasticity and beyond-microstructures, crystal-plasticity and phase transitions. CISM Courses and Lectures, Bd. 550. Springer, Berlin Heidelberg, S 1-64

21. Behrens B-A, Bouguecha A, Bonk C, Chugreev A (2017) Numerical and experimental investigations of the anisotropic transformation strains during martensitic transformation in a low alloy $\mathrm{Cr}$ Mo steel 42CrMo4. Proc Eng (207):1815-1820, https://doi.org/10. 1016/j.proeng.2017.10.944

22. Behrens B-A, Chugreev A, Kock C (2019) Macroscopic FE-simulation of residual stresses in thermo-mechanically processed steels considering phase transformation effects. XIV International Conference on Computational Plasticity. Fundamentals and Applications, S 211-222

23. Special Metals Co Special Metals Co, New Hartford, New York, USA. http://www.specialmetals.com/assets/smc/540documents/ pcc-8064-sm-alloy-handbook-v04.pdf. Zugegriffen: 8 Jan 2020

24. Behrens B-A, Chugreev A, Kock C (2018) Experimental-numerical approach to efficient TTT-generation for simulation of phase transformations in thermomechanical forming processes. IOP Conference Series, Materials Science and Engineering. Bd. 461

25. Acht C, Dalgic M, Frerichs F, Hunkel H, Irretier A, Lübben T, Surm H (2008) Ermittlung der Materialdaten zur Simulation des Durchhärtens von Komponenten aus 100Cr6. J Heat Treat Mater (63):234-244, https://doi.org/10.3139/105.100470 
26. Sente Software Ldt JMatPro. Practical software for materials properties. https://www.sentesoftware.co.uk/jmatpro. Zugegriffen: 8 . Jan. 2020

27. Uebing S, Brands D, Scheunemann L, Schröder J (2021) Residual stresses in hot bulk formed parts: two-scale approach for austenite-to-martensite phase transformation. Arch Appl Mech (91):545-562, https://doi.org/10.1007/s00419-020-01836-7
28. Uebing S, Brands D, Scheunemann L, Schröder J (2021) Residual stresses in hot bulk formed parts-microscopic stress analysis for austenite-to-martensite phase transformation. Arch Appl Mech. https://doi.org/10.1007/s00419-021-01921-5

29. Simo JC, Hughes TJR (1998) Computational inelasticity. Springer, Berlin

30. Stahl-Eisen-Prüfblätter 3rd Edition, 1990. 